cross-presentation ${ }^{9}$. Surprisingly, however, DNGR-1 and Syk were found ${ }^{9}$ not to be involved in the capture and engulfment of necrotic cells by the dendritic cell, implying that other cellular receptors are involved in this process.

Although it had been shown ${ }^{9}$ that DNGR-1 recognizes an intracellular DAMP that is exposed only after cell damage, it was unclear exactly which structure, or ligand, DNGR-1 binds to. Ahrens et al. ${ }^{3}$ and Zhang et al. ${ }^{4}$ managed to identify this ligand by a nifty piece of detective work. Both groups demonstrated that the ligand can be found in cells from many species, including those of insects, suggesting that it has been conserved over a long evolutionary period. However, attempts to detect the ligand using conventional biochemical approaches proved difficult and routinely yielded cytoskeletal components, which are normally considered to be contaminants in such procedures. It was only when the researchers used microscopy to examine cells that had been stained with a soluble fluorescent probe of DNGR-1, and saw fluorescence in the same position as the actin cytoskeleton, that they realized that the ligand was likely to be a component of this structure. Indeed, both groups subsequently demonstrated that DNGR-1 recognizes filamentous actin, or F-actin - the structural form of actin found in cells - but not its monomeric form, G-actin (Fig. 1).

Actin is a highly conserved globular protein found in all cells of eukaryotes - organisms that include animals, plants and fungi. It associates with numerous actin-binding proteins and serves multiple functions ranging from maintaining cell shape to facilitating cell division. In fact, both groups ${ }^{3,4}$ found that some of these actin-binding proteins, including $\alpha$-actinin and spectrin, enhance DNGR- 1 recognition of F-actin, presumably owing to stabilization and/or formation of the actin-filament complexes. How DNGR-1 recognizes F-actin is unclear, although Zhang et al. ${ }^{4}$ did identify a ligand-binding site on the receptor.

These discoveries have profound implications for our understanding of the mechanisms of dead-cell recognition. Arguably the most exciting insight is that the recognition of exposed F-actin in necrotic cells by DNGR-1 can directly induce antigen cross-presentation. As an abundant protein that is exposed only upon cell damage, F-actin makes an ideal DAMP. Furthermore, because actin release is known to correlate with tissue damage, it is likely that such a ubiquitous protein is also recognized by other PRRs. The ability of F-actin to drive cross-presentation of dead-cell antigens suggests that DNGR-1 could be involved in the development of certain autoimmune diseases and in immune responses to intracellular infections that cause cell damage. Indeed, DNGR-1 was recently shown ${ }^{10,11}$ to be essential for mounting protective $\mathrm{CD} 8^{+} \mathrm{T}$-cell responses during cell-damaging viral infections.
These studies will also enhance our understanding of the functions of the Syk signalling pathway used by CLRs. This pathway normally activates dendritic cells, triggering them to mature and to produce inflammatory mediators, as well as inducing their ability to activate the adaptive immune system. Unexpectedly, DNGR-1 does not use the signalling pathway in this way, and is not involved in driving inflammatory responses to necrotic cells $^{3,10}$. Rather, the DNGR-1 receptor seems to be responsible for diverting the necrotic-cell cargo ingested by dendritic cells to an intracellular pathway that favours cross-presentation ${ }^{10}$. Curiously, Syk-mediated signalling initiated by DNGR-1 is not required for this diversion, and yet it is essential for inducing cross-presentation ${ }^{10}$ (Fig. 1). How these cellular processes fit together remains a mystery, but the identification of F-actin as the DNGR-1 ligand should help us to decipher these essential mechanisms..

Gordon D. Brown is at the Institute of

Medical Sciences, University of Aberdeen,

Aberdeen AB25 2ZD, UK.

e-mail:gordon.brown@abdn.ac.uk

1. Rock, K. L., Lai, J. J. \& Kono, H. Immunol. Rev. 243, 191-205 (2011).

2. Seong, S. Y. \& Matzinger, P. Nature Rev. Immunol. 4, 469-478 (2004).

3. Ahrens, S. et al. Immunity $\mathbf{3 6}$, 635-645 (2012).

4. Zhang, J. G. et al. Immunity 36, 646-657 (2012).

5. Osorio, C. \& Reis e Sousa, C. Immunity 34, 651-664 (2011).

6. Sancho, D. et al. J. Clin. Invest. 118, 2098-2110 (2008).

7. Huysamen, C. et al. J. Biol. Chem. 283, 16693-16701 (2008).

8. Caminschi, l. et al. Blood 112, 3264-3273 (2008).

9. Sancho, D. et al. Nature 458, 899-903 (2009).

10.Zelenay, S. et al. J. Clin. Invest. 122, 1615-1627 (2012).

11.Iborra, S. et al. J. Clin. Invest. 122, 1628-1643 (2012).

\title{
CANCER METABOLISM
}

\section{Tumour friend or foe}

\section{Elucidation of a signalling pathway that promotes tumour-cell survival during metabolic stress reveals that a protein called AMPK may both hinder and enhance cancer progression. SEE LETTER P.661}

\section{ROBERT U. SVENSSON \& REUBEN J. SHAW}

$\mathrm{T}$ Tumour cells face various metabolic stresses as they arise and progress. Early-stage tumours that have not yet recruited new blood vessels to supply them with nutrients will be short of both glucose and oxygen $^{1}$, and some cells face metabolic stress when they make the transition from a normal to a cancerous state and become detached from the extracellular matrix, their normal environment $^{2}$. Additionally, some mutations that trigger tumour formation accelerate certain cellular metabolic programs and thereby cause stress. A protein called AMP-activated protein kinase (AMPK) is a central sensor of cellular metabolism. It is activated during situations of metabolic stress that lower the intracellular levels of ATP, a crucial energy-supplying molecule $^{3}$. On page 661 of this issue, Jeon et al. report ${ }^{4}$ a mechanism by which AMPK dictates tumour-cell survival, and the surprising implication that it may have a cancer-promoting role in some contexts.

The protein that phosphorylates and activates AMPK under conditions of metabolic stress is encoded by a gene called $L K B 1$, which is classed as a tumour suppressor: mutations that inactivate this gene are among the most common causes of human lung cancer. However, the LKB1 protein phosphorylates 12 kinase proteins in addition to AMPK, and it is not clear whether LKB1's tumour-suppressor function lies in its ability to activate AMPK, some of these other kinases, or both. What is clear is that AMPK activation influences cellular signalling pathways that are involved in cancer, such as the mTOR and p53 pathways, and inhibits the proliferation of cells in culture ${ }^{3,5}$. LKB1-dependent activation of AMPK also acts as a general metabolic 'rheostat': under conditions of metabolic stress, this activation directs the reprogramming of many cellular metabolic processes ${ }^{5-7}$ to restore energy homeostasis, which is essential for cell survival. But it is also not known which of AMPK's target proteins are most important for this activity.

Jeon et al. provide compelling evidence that AMPK exerts its effect on cell survival by regulating cellular levels of NADPH, a coenzyme that is essential for many biosynthetic reactions and which helps to remove dangerous reactive oxygen species (ROS) from cells (Fig. 1). The researchers show that AMPK indirectly regulates NADPH by inhibiting the metabolic enzymes acetyl-CoA carboxylase 1 (ACC1) and ACC2, the first substrates of AMPK to be discovered ${ }^{8}$. These enzymes control both fatty-acid synthesis, a process that consumes NADPH, and fatty-acid oxidation, which leads to NADPH regeneration.

Jeon and colleagues also demonstrate that control of the ACC enzymes and NADPH by AMPK is required for the survival of glucose-deprived cells, as well as for that of cells 
detached from the extracellular matrix. These data fit nicely with the findings of a previous study ${ }^{2}$ that demonstrated that the survival of detached mammary epithelial cells depends on NADPH and the neutralization of cellular ROS. The new results also agree with the finding ${ }^{9}$ that the death of tumour cells during glucose deprivation is reduced by AMPK activation and fattyacid oxidation. Although Jeon and colleagues have demonstrated a major role for NADPH in AMPK-dependent survival ${ }^{4}$, improved molecular tracing methods will be needed in future studies to determine quantitatively where in a cell NADPH is produced and consumed during the various forms of metabolic stress, and the extent to which production or consumption is influenced by ACC or AMPK.

In addition to its role in conditions of low nutrient levels or cell detachment, as observed by Jeon et al., AMPK may be required to maintain metabolic homeostasis in situations of deregulated, 'revved-up' metabolism that occur $^{10}$ in response to the activity of oncogenes - genes that promote tumorigenesis. One oncogene implicated in many human cancers is $c-M Y C$, which encodes a transcription factor, MYC, that primes cells for proliferation by expanding their biosynthetic capacity and reprogramming their metabolism. A recent study ${ }^{10}$ found that MYC-induced metabolic acceleration results in the activation of AMPK's function as a metabolic regulator and that AMPK and one of its related kinases (ARK5) are required for cell survival, but only under the conditions of elevated MYC levels. Another recent study ${ }^{11}$ screened more than 200 metabolism-associated genes for effects on cell viability and found that a gene encoding a subunit of AMPK is one of only two genes for which decreased expression resulted in reduced survival of prostate-cancer cells, but had no effect on normal prostate cells.

This selective death of cancer cells in response to reduced AMPK begs the question of whether the protein normally has a protumorigenic effect or whether it more often acts to keep cancer at bay. AMPK is known to exert antiproliferative effects by suppressing many oncogenic signalling pathways and biosynthetic processes, and it is thought to be a major mediator of the tumour-suppressive effect of LKB1 (ref. 3). So how might these previously observed anticancer effects be reconciled with the idea that AMPK function permits survival of tumour cells? Is it possible that the timing or cell-type specificity of the loss of AMPK activity is pivotal in determining whether this event halts tumours or promotes their success by 'rewiring' growth and metabolic pathways to favour cell proliferation?

This potential dual role for AMPK in tumours parallels the current view of the role of autophagy in cancer. Autophagy is a process by which cells break down and recycle their own components, and is one of the metabolic

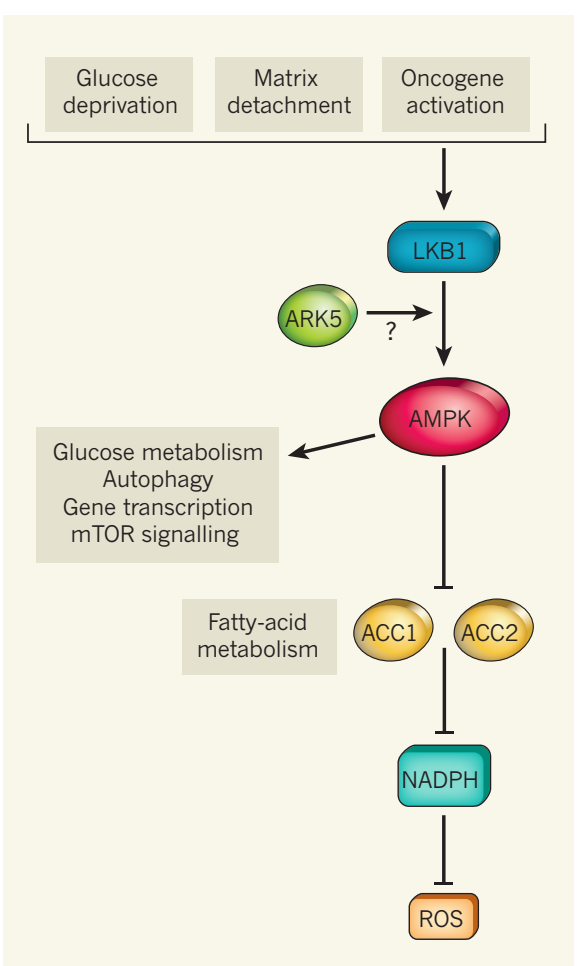

Figure 1 | Promoting cancer-cell survival during metabolic stress. The signalling pathway that involves the kinase proteins LKB1 and AMPK is activated in many tumour cells during conditions of metabolic stress, such as glucose deprivation and detachment from the normal extracellular matrix, and in response to the activation of some oncogenes. The pathway influences many metabolic and cancer-related processes, including glucose metabolism, autophagy, gene transcription and mTOR signalling. Jeon et al. ${ }^{4}$ show that the pathway can promote tumour-cell survival by inhibiting two AMPK-regulated enzymes, ACC1 and ACC2, which are involved in fatty-acid metabolism. These enzymes lower cellular levels of the coenzyme $\mathrm{NADPH}$, which acts to attenuate dangerous reactive oxygen species (ROS) that accumulate during metabolic stress. It has also been recently demonstrated ${ }^{10}$ that the AMPK-related kinase protein ARK5 is involved in cell survival in response to activated oncogenes in some settings.

systems regulated by AMPK. In some cases, inhibition of autophagy promotes tumour initiation, but in many other contexts autophagy is needed to keep later-stage tumours alive, such that its inhibition might be expected to have an anticancer effect ${ }^{12}$. Indeed, Jeon $e t$ al. demonstrate that reducing LKB1 or AMPK expression in a mouse model of breast cancer significantly inhibits the growth of mammarytumour cells, and that the presence of modified versions of ACC1 and ACC2 that cannot be phosphorylated by AMPK also leads to reduced tumour growth. Such findings suggest that these effects result from inhibition of the survival-promoting pathways that stem from ACC1 and ACC2. However, the opposite effect has been observed when $L K B 1$ is knocked out as an early event, including in some mouse models in which cancer development is driven by the activation of Myc (ref. 13) or another oncogenic protein, Kras (ref. 14 ) - here, loss of LKB1 strongly accelerates tumour growth.

How can these paradoxical results be resolved? It is possible that cultured tumour cells have a greater need for AMPK for survival than do tumours in vivo because nutrients in cell culture media are exhausted more rapidly than nutrients in an intact animal. Alternatively, the difference might arise from the timing of these events, and therefore depend on whether LKB1 or AMPK is inactivated in early- or late-stage tumours. This situation might explain previous data showing ${ }^{15,16}$ that LKB1 - and AMPK-deficient cells are unexpectedly resistant to artificial transformation from a normal to a cancerous state in vitro, although this observation might also relate to altered metabolic demands in cell culture. Interestingly, another context in which AMPK activation seems to have both tumour-suppressing and tumour-promoting effects was recently reported ${ }^{17}$ in melanoma cells expressing the oncogene $B R A F$.

Only time and a more thorough dissection of these possibilities in different cell types and mouse models will reveal what determines the part AMPK plays in different cancers. In the meantime, the prospect that AMPK inhibition might sensitize advanced tumour cells to chemotherapy, or even to their own deregulated metabolic demands, warrants further examination. AMPK may therefore be both friend and foe to cancer, depending on when and why it is needed.

Robert U. Svensson and Reuben J. Shaw are at the Molecular and Cell Biology Laboratory, Howard Hughes Medical Institute, The Salk Institute for Biological Studies, La Jolla, California 92037, USA.

e-mail:shaw@salk.edu

1. Hanahan, D. \& Weinberg, R. A. Cell 144, 646-674 (2011).

2. Schafer, Z. T. et al. Nature 461, 109-113 (2009)

3. Hardie, D. G., Ross, F. A. \& Hawley, S. A. Nature Rev. Mol. Cell Biol. 13, 251-262 (2012).

4. Jeon, S.-M., Chandel, N. S. \& Hay, N. Nature 485, 661-665 (2012).

5. Mihaylova, M. M. \& Shaw, R. J. Nature Cell Biol. 13, 1016-1023 (2011)

6. Egan, D. F. et al. Science 331, 456-461 (2011).

7. Gwinn, D. M. et al. Mol. Cell 30, 214-226 (2008).

8. Carling, D., Clarke, P. R., Zammit, V. A. \& Hardie, D. G. Eur. J. Biochem. 186, 129-136 (1989).

9. Buzzai, M. et al. Oncogene 24, 4165-4173 (2005).

10.Liu, L. et al. Nature 483, 608-612 (2012).

11.Ros, S. et al. Cancer Discov. 2, 328-343 (2012).

12. Mathew, R. \& White, E. Curr. Opin. Genet. Dev. 21, 113-119 (2011).

13.Partanen, J. I. et al. Proc. Natl Acad. Sci. USA 109, E388-E397 (2012)

14.Ji, H. et al. Nature 448, 807-810 (2007).

15.Bardeesy, N. et al. Nature 419, 162-167 (2002).

16. Laderoute, K. R. et al. Mol. Cell. Biol. 26, 5336-5347 (2006).

17.Martin, M. J., Hayward, R., Viros, A. \& Marais, R. Cancer Discov. 2, 344-355 (2012) 